\title{
Transseptal approach is preferred for transcatheter mitral valve-in-valve procedures
}

\author{
John J. Squiers ${ }^{1}$, J. James Edelman ${ }^{2}$, Vinod H. Thourani ${ }^{3}$, Michael J. Mack ${ }^{1,4}$ \\ ${ }^{1}$ Baylor Scott \& White Research Institute, Plano, Texas, USA; ${ }^{2}$ Fiona Stanley Hospital, University of Western Australia, Perth, Australia; \\ ${ }^{3}$ Department of Cardiovascular Surgery, Marcus Valve Center, Piedmont Heart Institute, Atlanta, GA, USA; ${ }^{4}$ Department of Cardiothoracic Surgery, \\ Baylor Scott \& White, The Heart Hospital, Plano, Texas, USA \\ Correspondence to: Michael J. Mack, MD. Baylor Scott and White Health Heart Hospital-Plano, 1100 Allied Dr., Plano, TX 75093, USA.. Email: \\ michael.mack@bswhealth.org.
}

Submitted Jun 13, 2021. Accepted for publication Jul 14, 2021.

doi: 10.21037/acs-2021-tviv-23

View this article at: https://dx.doi.org/10.21037/acs-2021-tviv-23

The use of bioprosthetic valves for mitral valve (MV) replacement (MVR) has increased from less than $20 \%$ to greater than $75 \%$ of cases over the last twenty-five years $(1,2)$. It is unsurprising that bioprosthetic $M V$ degeneration requiring re-intervention is becoming more commonplace. Given that re-operative MV surgery carries a significant $10-15 \%$ risk of peri-operative mortality as ascertained by the Society of Thoracic Surgeons' (STS) database (3), transcatheter MVR via a valve-in-valve $(\mathrm{MViV})$ technique has been investigated as an alternative approach for high- and extreme-risk patients with degenerated mitral bioprostheses. Early experience with MViV focused on the transapical (TA) approach (4). Although the TA approach was initially favored because it is less technically challenging (5), $\mathrm{MViV}$ has become increasingly performed via a transseptal (TS) approach.

The most comprehensive outcome data regarding $\mathrm{MViV}$ procedures comes from three registries: the Valvein-Valve International Data (VIVID) registry (5), the transcatheter mitral valve replacement (TMVR) registry (6), and the STS/American College of Cardiology (ACC) Transcatheter Valve Therapies (TVT) registry (7), all of which have published recent updates on their experiences with $M V i V$. In all three registries, technical success for MViV exceeded $90 \%$ with both TA and TS approaches. No differences for in-hospital mortality were identified when comparing approach techniques in any of these analyses, though the TVT registry report identified a trend towards lower in-hospital mortality with TS, as compared to the TA approach $(\mathrm{P}=0.06)$. Regarding peri-procedural morbidity, there were no differences identified in the VIVID registry (6). The TMVR registry showed higher rates of atrial septal defect closure and lower rates of lifethreatening or fatal bleeding events after TS access (7). The TVT registry analysis demonstrated shorter lengths of stay and increased rates of discharge to home among patients undergoing TS as compared to TA MViV, despite no statistically significant differences in rates of complications including stroke, re-intervention, need for new permanent pacemaker, or bleeding (8).

The TS approach is substantially less invasive than the $\mathrm{TA}$ approach. Therefore, $\mathrm{TS} \mathrm{MViV}$ is expected to decrease peri-procedural risk for patients, especially because cardiac teams are achieving equivalent technical success despite the increased procedural complexity of this technique. Why then does the most comprehensive available data provide no evidence of superior in-hospital survival after TS MViV and only very limited evidence regarding reduced morbidity associated with this technique? First, it is essential to recognize that these registries include a limited number of cases for evaluation (VIVID N=857; TMVR N=322; and TVT N=1,529). Secondly, the VIVID and TMVR registry reports include cases performed over a substantial time frame (VIVID 2006-2020; and TMVR 2009-2018) that likely captured the early experience and learning curve for the TS approach, which may have exceeded that of the TA approach due to prior experience with TA TAVR. The TVT report, in which trends favoring TS access began to emerge, comprises of data from 2015 to 2019, when the TS learning curve had likely been overcome by most participating centers. Therefore, the absence of data demonstrating a reasonably expected 
advantage peri-procedural outcomes with respect to the TS approach might be explained by a lack of statistical power to demonstrate differences, a learning curve during the early experience of TS MViV belying superior outcomes, or a combination of these factors.

Most importantly, while each of these registries document only a $30-40 \%$ utilization of the TS approach, this data ultimately reflects the evident bias towards the TA approach early in the development of MViV (6-8). Both the VIVID (6) and TVT (8) registry MViV reports, as well as a global update on all transcatheter MV therapies from the TVT registry (9), demonstrate significant trends regarding the adoption of the TS approach for $\mathrm{MViV}$, increasing from $<16 \%$ during 2006-2013 to $>80 \%$ during 2018-2020. Even accounting for the dearth of peer-reviewed evidence regarding peri-procedural differences among MViV approaches, this trend unequivocally demonstrates which access is preferred by structural cardiac teams internationally. Paralleling this shift, operative mortality during MViV as reported by the TVT registry, declined from $11.1 \%$ in 2014 to $6.2 \%$ in 2019 (9). Though no comparison between operative mortality after TA versus TS MViV was performed alongside these data outcomes, clinical practice is clearly demonstrating what the peer-reviewed literature has yet to recognize.

A substantial gap in the current literature is the limited comparative data available on long-term outcomes after MViV. For the first time, the TVT registry has recently demonstrated superior one-year survival after TS versus TA access for MViV (8). Although outcome data beyond the first year is still necessary, there can be no long-term follow-up without first achieving short-term success. The evidence we do have suggests that there are, at minimum, no differences in peri-procedural outcomes for the TA and TS approaches-and a tie goes to the less invasive approach. Trends are emerging in the most recent data that favor TS access, and as we have outlined, we suspect that as additional experience and evidence accumulates, the advantages of TS access will become readily apparent. Until then, MV cardiac teams with limited TS experience should pursue additional training and proctoring for this technique, and all structural cardiac proceduralists should continue to work to improve outcomes of $\mathrm{MViV}$ by all approaches.

\section{Acknowledgments}

Funding: None.

\section{Footnote}

Conflicts of Interest: Dr. MJM is a trial investigator for Edwards Lifesciences, Medtronic and Abbott; Dr. VHT is an advisor for Edwards Lifesciences. The other authors have no conflicts of interest to declare.

Open Access Statement: This is an Open Access article distributed in accordance with the Creative Commons Attribution-NonCommercial-NoDerivs 4.0 International License (CC BY-NC-ND 4.0), which permits the noncommercial replication and distribution of the article with the strict proviso that no changes or edits are made and the original work is properly cited (including links to both the formal publication through the relevant DOI and the license). See: https://creativecommons.org/licenses/by-nc-nd/4.0/.

\section{References}

1. Goldstone AB, Chiu P, Baiocchi M, et al. Mechanical or Biologic Prostheses for Aortic-Valve and Mitral-Valve Replacement. N Engl J Med 2017;377:1847-57.

2. Gammie JS, Chikwe J, Badhwar V, et al. Isolated Mitral Valve Surgery: The Society of Thoracic Surgeons Adult Cardiac Surgery Database Analysis. Ann Thorac Surg 2018;106:716-27.

3. Mehaffey HJ, Hawkins RB, Schubert S, et al. Contemporary outcomes in reoperative mitral valve surgery. Heart 2018;104:652-6.

4. Seiffert M, Conradi L, Baldus S, et al. Transcatheter mitral valve-in-valve implantation in patients with degenerated bioprostheses. JACC Cardiovasc Interv 2012;5:341-9.

5. Simonato $\mathrm{M}$, Whisenant $\mathrm{B}$, Ribeiro $\mathrm{HB}$, et al. Transcatheter Mitral Valve Replacement After Surgical Repair or Replacement: Comprehensive Midterm Evaluation of Valve-in-Valve and Valve-in-Ring Implantation From the VIVID Registry. Circulation 2021;143:104-16.

6. Yoon SH, Whisenant BK, Bleiziffer S, et al. Outcomes of transcatheter mitral valve replacement for degenerated bioprostheses, failed annuloplasty rings, and mitral annular calcification. Eur Heart J 2019;40:441-51.

7. Whisenant B, Kapadia SR, Eleid MF, et al. One-Year Outcomes of Mitral Valve-in-Valve Using the SAPIEN 3 Transcatheter Heart Valve. JAMA Cardiol 2020;5:1245-52.

8. Guerrero M, Vemulapalli S, Xiang Q, et al. Thirty-Day Outcomes of Transcatheter Mitral Valve Replacement for Degenerated Mitral Bioprostheses (Valve-in-Valve), 
Failed Surgical Rings (Valve-in-Ring), and Native Valve With Severe Mitral Annular Calcification (Valve-in-Mitral Annular Calcification) in the United States: Data From the Society of Thoracic Surgeons/American College of Cardiology/Transcatheter Valve Therapy Registry. Circ

Cite this article as: Squiers JJ, Edelman JJ, Thourani VH, Mack MJ. Transseptal approach is preferred for transcatheter mitral valve-in-valve procedures. Ann Cardiothorac Surg 2021;10(5):697-699. doi: 10.21037/acs-2021-tviv-23
Cardiovasc Interv 2020;13:e008425.

9. Mack MJ, Carroll JD, Thourani V, et al. Transcatheter mitral valve therapy in the US: A 2020 report from the STS-ACC TVT Registry. J Am Coll Cardiol 2021. [In press]. 